\title{
The Formation of Tidal Dwarf Galaxies in Interacting Systems: the Case of Arp 245 (NGC 2992/93)
}

\author{
Elias Brinks \\ Depto. de Astronomía, Univ. de Guanajuato, Apdo. Postal 144, Guanajuato, \\ Mexico \\ Pierre-Alain Duc \\ CNRS and CEA-Saclay, Service d'Astrophysique, France \\ Volker Springel \\ Center for Astrophysics, Cambridge, MA, USA \\ Barbara Pichardo \\ Instituto de Astronomía, UNAM, Mexico DF, Mexico \\ Peter Weilbacher \\ Universitätssternwarte, Göttingen, Germany \\ Felix Mirabel \\ CEA-Saclay, Service d'Astrophysique, France and IAFE, Buenos Aires, Argentina
}

\begin{abstract}
.
We present some highlights of our multi-wavelength study, which involves optical broad- and narrow-band imaging, long-slit spectroscopy, high-resolution H I, and $\mathrm{CO}$ observations, of the interacting system Arp 245. This object consists of the galaxies NGC 2992 and NGC 2993. Based on a numerical model of the collision, which was computed with a Tree-SPH code, we derive that Arp 245 is observed at an early stage of the interaction, about $100 \mathrm{Myr}$ after perigalacticon, though at a time when tidal tails have already developed. At the tip of the NGC 2992 tail we find a gas reservoir of about $10^{9} \mathrm{M}_{\odot}$, or about $60 \%$ of the $\mathrm{HI}$ which is seen towards NGC 2992, which coincides with what appears to be a star-forming tidal dwarf galaxy, A245N. The TDG A245N exhibits properties ranging between those of dwarf irregular galaxies (structural parameters, gas content, star formation rate) and those of spiral disks (metallicity, star formation efficiency, stellar population). We speculate what the required conditions are to form a TDG, and how they can be distinguished from field dwarf irregulars.
\end{abstract}

Keywords: galaxies: interactions — galaxies: individual (NGC 2992, NGC 2993, FGC 0938) — radio emission lines: H I

\section{Introduction}

At larger look-back times, interactions between galaxies are thought to have been more frequent, ejecting stellar and gaseous material into the intergalactic medium as a result of strong tidal forces. In the local Universe, these processes can be studied in much more detail of course, 


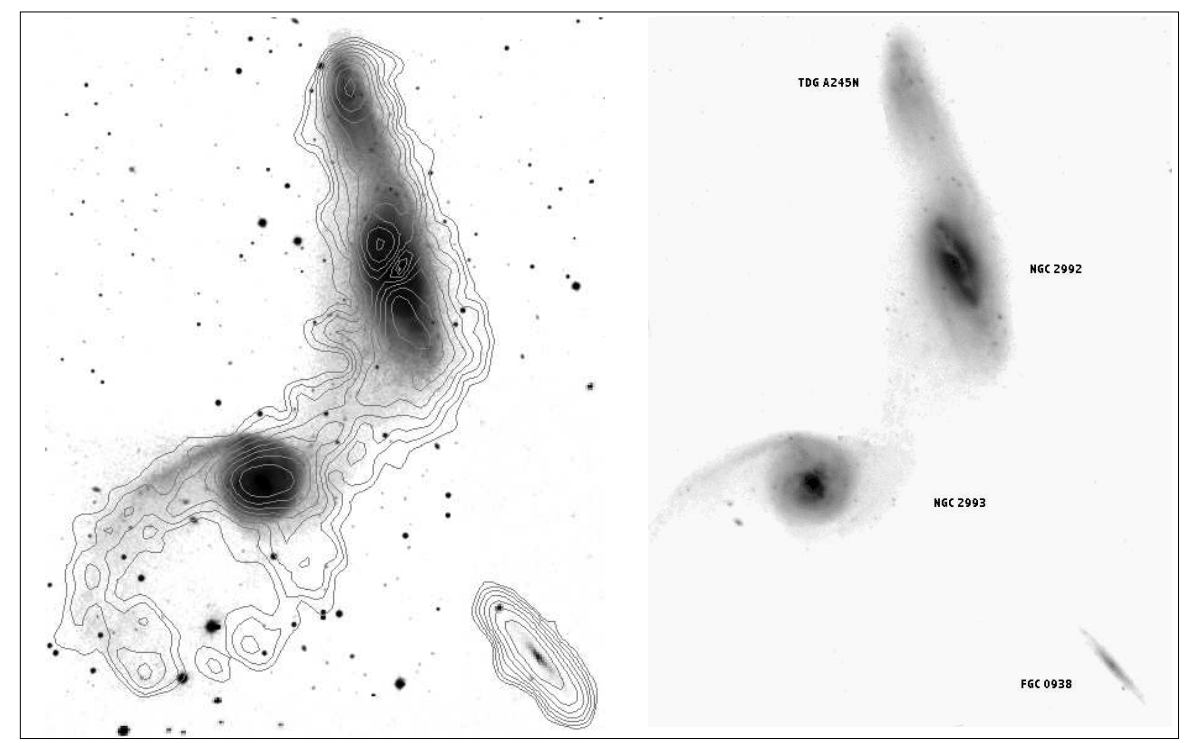

Figure 1. Left: Optical V-band CCD image of the interacting system Arp 245 with overlayed VLA H i contours. Right: Same V-band image with labels identifying the different objects that can be recognised.

and we find that under certain conditions ejected tidal material can recollect and collapse to form tidal dwarf galaxies (TDGs).

After having been postulated by Zwicky (1956), Schweizer (1978) was the first one to tentatively identify dwarf galaxies which had been formed out of the debris of a galaxy interaction. This new class of objects is enjoying a great deal of interest after the seminal paper by Mirabel et al. (1992). A review of the field can be found in Duc \& Mirabel (1999).

In brief, a tidal dwarf galaxy can be characterized as follows. It is a self-gravitating collection of stars and gas which has formed out of the debris of a tidal interaction between galaxies, at least one of which was gas-rich. They are usually found near the tips of tidal arms. Recent star formation is taking place in situ, the travel time for gas and stars to move from the parent galaxies to the location of the TDGs being several times larger than the age of the star forming regions. Recently, molecular gas has been detected by Braine et al. (2000) towards the H II regions of several TDGs. 


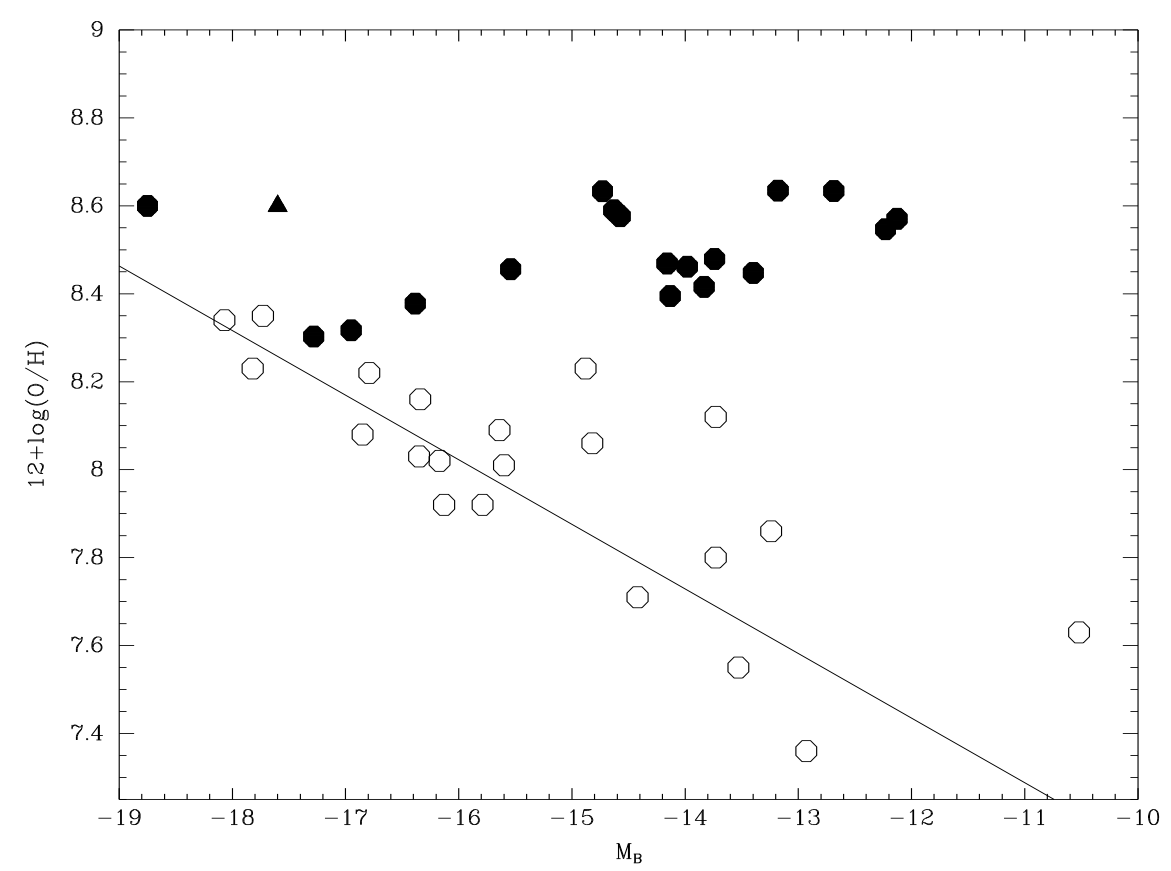

Figure 2. Oxygen abundance versus absolute blue magnitude for a sample of isolated nearby dwarf galaxies (open circles; Richer \& McCall 1995), and tidal dwarf galaxies (filled circles; Duc \& Mirabel 1998). The tidal dwarf A245N is indicated by a triangle.

\section{Arp 245 (NGC2992/93)}

Figure 1 shows in the right-hand panel an optical CCD optical image obtained at the ESO NTT telescope. Labels are shown to identify the various objects. At the tip of the pronounced, $30 \mathrm{kpc}$ long tail of NGC 2992 we find a concentration of stars and regions of recent star formation. On the left, at the same scale, we show again the V-band image, overlayed with $\mathrm{H}$ I contours. The H I observations were obtained with the $\mathrm{NRAO}^{1}$ Very Large Array. More details on the observations can be found in Duc et al. (2000).

\section{Discussion}

TDGs exhibit properties that range between those of dwarf irregular galaxies (structural parameters, gas content, star formation rate) and

1 The National Radio Astronomy Observatory is a facility of the National Science Foundation operated under cooperative agreement by Associated Universities, Inc. 
those of spiral disks (metallicity, star formation efficiency, stellar population). It is because of the duality of their nature that one can, in principle, distinguish between field dwarfs and TDGs, even if signs of a previous interaction have long since disappeared. A powerful diagnostic is the heavy element abundance (metallicity) of TDGs which tends to cluster around a constant value of $1 / 3$ of solar. This is roughly the value encountered in the outskirts of spiral galaxies which is where most of the gas which gets torn into tidal tails originates. Figure 2 compares the metallicity-luminosity relation known for field dwarf galaxies with that for TDGs. The corresponding value for TDG A245N as determined by Duc et al. (2000) is indicated by a filled triangle.

Another approach depends on the hypothesis that TDGs contain less than 5\% dark matter (Barnes \& Hernquist 1992). Unfortunately, no observations with high enough sensitivity and/or velocity resolution, be it in the optical or in the radio, have been reported to date to confirm or refute this hypothesis as a useful tool to distinguish a TDG from a field dwarf.

Other possible approaches towards determining if an object has a tidal origin have been listed by Hunter et al. (2000), such as their morphology and the make-up of their stellar population, which should be bimodal, a fraction of the older stars having come from one of the parent galaxies and having combined with recently formed stars created out of the gas which has collected at or near the tip of the tidal tail.

Several projects are underway to search for tidal dwarf galaxy candidates (Weilbacher et al., this volume; Iyer et al., this volume) which eventually should increase our knowledge regarding this type of objects.

\section{References}

Barnes, J.E., \& Hernquist, L. 1992, Nature, 360, 715

Braine, J., Lisenfeld, U., Duc, P.-A., \& Leon, S. 2000, Nature, 403, 867

Duc, P.-A., Brinks, E., Springel, V., Pichardo, B., Weilbacher, P. \& Mirabel, I.F. 2000, AJ (in press)

Duc, P.-A., \& Mirabel, I.F. 1998, A\&A, 333, 813

Duc, P.-A., \& Mirabel, I.F. 1999, in IAU Symp. No. 186: Galaxy Interactions at Low and High Redshift, eds. J.E. Barnes \& D.B. Sanders (Kluwer: Dordrecht), p. 61

Hunter, D.A., Hunsberger, S.D., \& Roye, E.W. 2000, ApJ (in press) astro-ph/0005257

Mirabel, I.F., Dottori, H., \& Lutz, D. 1992, A\&A, 256, L19

Richer, M.G., \& McCall, M.L. 1995, ApJ, 445, 642

Schweizer, F. 1978 in IAU Symp. No. 77: Structure and properties of nearby galaxies, eds. E.M. Berkhuijsen \& R. Wielebinski (Reidel: Dordrecht), p. 279

Zwicky, F. 1956, in Ergebnisse der Exakten Naturwissenschaften, 29, 344 